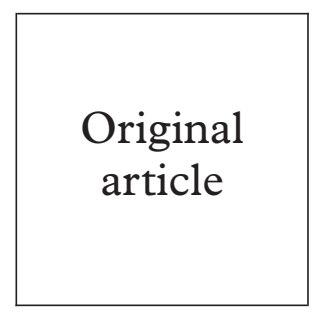

\title{
Syndromic management of urethral discharge in Ghanaian pharmacies
}

\author{
Y Adu-Sarkodie, M J Steiner, J Attafuah, K Tweedy
}

Objectives: To evaluate the training of pharmacists in Accra, Ghana, in the syndromic management of STIs.

Methods: We randomly selected 50 pharmacy outlets that had received the training (intervention) and 50 outlets that had not received the training (no intervention). Simulated clients described the symptoms of urethral discharge to the first pharmacy staff encountered and completed a standardised questionnaire after each encounter.

Results: Correct drug provision for urethral discharge improved with the educational intervention but remained relatively low (no intervention 18\%; intervention 39\%; p <0.05). More encouraging, treatment for gonorrhoea was usually correct without the intervention (64\%) and improved further in the intervention outlets $(76 \%)$. The treatment for chlamydia was less often appropriate but also improved (31\% and $41 \%)$. Condom promotion was poor, with almost no outlets offering condoms.

Conclusions: The current training led to improvements in the treatment of urethral discharge. Future training needs to be improved, especially with regard to condom promotion. Moreover, since less than one third of simulated clients were seen by pharmacists, the training should be expanded to other pharmacy staff. With enhanced training of all pharmacy staff, the role of pharmacy outlets in STI management and prevention in Ghana and elsewhere can be optimised. (Sex Transm Inf 2000;76:439-442)

Keywords: pharmacists; sexually transmitted infections; syndromic management

\section{Introduction}

The Mwanza study ${ }^{1}$ documented a decrease in the incidence of HIV infection as a result of modest community improvement in the management of sexually transmitted infections (STIs). In Ghana, the ministry of health $(\mathrm{MOH})$ began training healthcare workers in the syndromic management of STIs, with the early efforts focused mainly on clinicians in the public sector. Subsequent research by the $\mathrm{MOH}$ indicated that the predominant providers of STI care in Ghana are pharmacists. ${ }^{2}$

Three recent approaches to train pharmacists in the syndromic management of STIs have documented varying levels of success. ${ }^{3-5}$ In Cameroon, a pilot project to social market urethritis treatment packages containing antibiotics, condoms, partner referral cards, and written information through private pharmacies fell short of expectations when opposition from the local medical community forced a scaled down introduction limited to primary healthcare facilities. ${ }^{3}$ In Peru, a randomised study to evaluate an educational intervention at pharmacies found only a modest improvement in STI management. ${ }^{4}$ Intervention pharmacies provided counselling more often than did controls $(40 \% v 27 \%, \mathrm{p}=0.01)$; however, few of the simulated clients received the correct drug $(15.3 \% \vee 15.7 \%)$ and fewer still received the correct regimen $(1.4 \% v 0.7 \%)$. More encouraging, a simulated client study in Nepal documented an increase in the provision of the correct $\mathrm{drug} / \mathrm{regimen}$ from $0.8 \%$ before the intervention to $45 \%$ immediately after the intervention. ${ }^{5}$
Based on $\mathrm{MOH}$ recommendations the training of pharmacists in syndromic management of STIs began in Accra with support from the National AIDS Control Programme (NACP) and funding from the West Africa Project to Combat AIDS and STD (WAPTCA). ${ }^{6} \mathrm{Be}-$ tween April 1997 and June 1998, 276 pharmacists had attended a 1 day training course which covered history taking and examination, therapy, condom promotion, partner notification, health education and counselling, and STI record keeping using the syndromic approach. This report evaluates the impact of this training.

\section{Methods}

We conducted a simulated client study in January 1999 at 100 pharmacies and chemist shops in Accra randomly selected from a sampling frame based on a research international (RI) merchandising study of all such outlets in Accra $(n=259)$. Although in rural areas of Ghana, STI drug provision may occur through the informal sector, in Accra the informal sector is believed to play at most a minor part in STI drug provision. We drew 50 outlets in Accra with staff who had received the STI training (intervention) and 50 outlets in Accra that had not received STI training (no intervention). We trained 10 male study staff to visit the selected outlets and describe to the first provider they encountered their symptoms of urethral discharge. To support the realism of their presentations, study staff reviewed a conversation guideline outlining different scenarios. Simulated clients were provided with 
Table 1 Background characteristics and counselling

\begin{tabular}{|c|c|c|}
\hline & $\begin{array}{l}\text { Accra, intervention } \\
(n=48)\end{array}$ & $\begin{array}{l}\text { Accra, no intervention } \\
(n=48)\end{array}$ \\
\hline & No $(\%)$ & No $(\%)$ \\
\hline \multicolumn{3}{|l|}{ Sex of staff: } \\
\hline Male & $27(56)$ & $39(81)$ \\
\hline Female & $21(44)$ & $9(19)$ \\
\hline \multicolumn{3}{|l|}{ Staff position: } \\
\hline Pharmacist & $14(29)$ & $13(27)$ \\
\hline Store clerk & $24(50)$ & $19(40)$ \\
\hline Don't know & $7(15)$ & $11(23)$ \\
\hline Other & $3(6)$ & $5(10)$ \\
\hline \multicolumn{3}{|l|}{ Number of staff at outlet: } \\
\hline 1 & $8(17)$ & $13(27)$ \\
\hline 2 & $19(40)$ & $20(42)$ \\
\hline 3 & $12(25)$ & $13(27)$ \\
\hline 4 & $8(17)$ & $1(2)$ \\
\hline $5+$ & $1(2)$ & $1(2)$ \\
\hline Asked details about discharge $\dagger$ & $28(58)$ & $24(50)$ \\
\hline Asked when discharge first noticed $\dagger$ & $30(63)$ & $27(56)$ \\
\hline Asked who may have given them infectiont & $19(40)$ & $12(25)$ \\
\hline Asked about other recent sexual contact $\dagger$ & $10(21)$ & $8(17)$ \\
\hline Partner notification discussed $\dagger$ & $19^{\star}(40)$ & $10(21)$ \\
\hline Asked about past condom uset & $4(8)$ & $3(6)$ \\
\hline Counselled to use condomst & $0(0)$ & $6^{\star}(13)$ \\
\hline If counselled received condom instructions & $0(0)$ & $1(17)$ \\
\hline
\end{tabular}

$+\chi^{2}$ test for difference in two proportions; test done only where noted; ${ }^{\star} \mathrm{p}<0.05$.

sufficient funds to cover the cost of purchasing drugs and/or condoms that they were offered. To further minimise detection, we completed all simulated client visits within 2 weeks. After each encounter, the simulated client completed a standardised questionnaire documenting details about the interaction. The study was approved by the research ethics committee of the School of Medical Sciences, University of Science and Technology, Kumasi.

Table 2 Diagnosis and treatment

\begin{tabular}{|c|c|c|}
\hline & $\begin{array}{l}\text { Accra, intervention } \\
(n=48)\end{array}$ & $\begin{array}{l}\text { Accra, no intervention } \\
(n=48)\end{array}$ \\
\hline & No (\%) & No (\%) \\
\hline Given a diagnosis $†$ & $14(29)$ & $10(21)$ \\
\hline \multicolumn{3}{|l|}{ If yes, what diagnosis was given: $\sqrt{ }$} \\
\hline Gonorrhoea & $12(86)$ & $7(70)$ \\
\hline Chlamydia & $0(0)$ & $1(10)$ \\
\hline Urinary tract infection & $2(14)$ & $1(10)$ \\
\hline $\mathrm{VD} / \mathrm{STI}$ & $0(0)$ & $2(20)$ \\
\hline \multicolumn{3}{|l|}{ What advised to do about discharge: } \\
\hline Offered oral medication & $42(88)$ & $40(83)$ \\
\hline Offered injection & $11(23)$ & $13(27)$ \\
\hline Told to attend health clinic & $5(10)$ & $5(10)$ \\
\hline Told to speak to other pharmacy staff & $0(0)$ & $0(0)$ \\
\hline Told to go to different pharmacy & $0(0)$ & $0(0)$ \\
\hline \multirow{2}{*}{\multicolumn{3}{|c|}{ If offered oral medication, appropriateness of treatment $\ddagger$}} \\
\hline Urethral discharge: & & \\
\hline $\mathrm{MOH}$ correct drug/dose & $4(10)$ & $1(3)$ \\
\hline Expert opinion correct drug/dose & $7(17)$ & $4(10)$ \\
\hline Total correct drug dose $\dagger$ & $11(27)$ & $5(13)$ \\
\hline $\mathrm{MOH}$ correct drug/dose too high & $4(10)$ & $1(3)$ \\
\hline Expert opinion correct drug/dose too high & $1(2)$ & $1(3)$ \\
\hline Total acceptable drug provision $\dagger$ & $16^{\star}(39)$ & $7(18)$ \\
\hline \multicolumn{3}{|l|}{ Gonorrhoea: } \\
\hline $\mathrm{MOH}$ correct drug/dose & $13(32)$ & $8(21)$ \\
\hline Expert opinion correct drug/dose & $4(10)$ & $4(10)$ \\
\hline Correct drug/dose $†$ & $17(41)$ & $12(31)$ \\
\hline $\mathrm{MOH}$ correct drug/dose too high & $10(24)$ & $8(21)$ \\
\hline Expert opinion correct drug/dose too high & $4(10)$ & $5(13)$ \\
\hline Total acceptable drug provision $\dagger$ & $31(76)$ & $25(64)$ \\
\hline \multicolumn{3}{|l|}{ Chlamydia: } \\
\hline $\mathrm{MOH}$ correct drug/dose & $5(12)$ & $2(5)$ \\
\hline Expert opinion correct drug/dose & $8(20)$ & $7(18)$ \\
\hline Correct drug/doset & $13(32)$ & $9(23)$ \\
\hline $\mathrm{MOH}$ correct drug/dose too high & $4(10)$ & $3(8)$ \\
\hline Expert opinion correct drug/dose too high & $0(0)$ & $0(0)$ \\
\hline Total acceptable drug provision $†$ & $17(41)$ & $12(31)$ \\
\hline
\end{tabular}

$+\chi^{2}$ test for difference in two proportions; tests done only where noted; ${ }^{\star} \mathrm{p}<0.05$.

$\ddagger$ Two simulated clients offered oral medication had missing information on type of drug provided (intervention $\mathrm{n}=41$; no intervention $\mathrm{n}=39$ ).

$\$$ Multiple responses possible.
We evaluated whether counselling and treatment differed in the outlets in greater Accra where a pharmacist received training compared with the outlets where a pharmacist did not receive training. Where noted, we tested for differences in two proportions using EPI-INFO (version 6.04B). Sample size was based more on logistical and financial constraints than statistical considerations; this sample size provides $75 \%$ power to detect a significant difference in correct provision between any two groups if the proportion is 0.50 in one group and 0.75 in the other $($ alpha $=0.05)$.

In settings where diagnostic tools are unavailable, the current recommendation is to treat males with urethral discharge for both gonorrhoea and chlamydia. ${ }^{7}$ The drug regimen recommended by the Ghana $\mathrm{MOH}$ for gonorrhoea is one dose of ciprofloxacin $(500 \mathrm{mg})$ or ceftriaxone $(250 \mathrm{mg})$. For chlamydia the $\mathrm{MOH}$ recommends: (1) two daily doses of doxycyline $(100 \mathrm{mg}$ ) for 7 days; (2) four daily doses of tetracycline $(500 \mathrm{mg}$ ) for 7 days; or (3) four daily doses of erythromycin $(500 \mathrm{mg})$ for 7 days. $^{8}$ Additional drug regimens considered appropriate by the first author are: for gonorrhoea one daily dose of norfloxacin $(800 \mathrm{mg})$, perfloxacin $(800 \mathrm{mg})$, or azithromycin $(1 \mathrm{~g})$ for 1 day; and for chlamydia two daily doses of doxycycline $(100 \mathrm{mg})$ or four daily doses of tetracycline $(500 \mathrm{mg})$ for 5 days, or one daily dose of azithromycin ( $1 \mathrm{~g})$ for 1 day.

\section{Results}

Simulated clients visited 96 outlets of the 100 in the original sample. The remaining four could not be located or were closed. The majority of pharmacy staff encountered were male (intervention $56 \%$; no intervention $81 \%$ ) with less than one third identified by the simulated client as being a pharmacist $(29 \%$ and $27 \%$, respectively) (table 1 ).

Overall, counselling and history taking were better at the outlets that had received training, but few of these differences were statistically significant. Over half the clients $(58 \%)$ at intervention outlets were asked details about their discharge ( $v 50 \%$ no intervention), while a slightly higher proportion of the intervention clients $(63 \%)$ were asked when the discharge was first noted (v 56\%). Similar differences were noted for being asked who may have given them the infection ( $40 \% v 25 \%$ ), recent sexual contacts $(21 \% v 17 \%)$, partner notification $(40 \% v 21 \%, \mathrm{p}<0.05)$, and past condom use $(8 \% v 6 \%)$. Despite condoms being widely available at pharmacies, no simulated client attending an intervention outlet was counselled to use condoms $(0 \% v 13 \%, \mathrm{p}<0.05)$.

About a quarter of simulated clients were given a diagnosis (intervention 29\%; no intervention $21 \%$ ) with the most common diagnosis being gonorrhoea $(86 \%$ and $70 \%$, respectively) (table 2). Most simulated clients were offered oral medications $(88 \%$ and $83 \%$, respectively) while about one quarter were offered injections $(23 \%$ and $27 \%$, respectively).

Few of the simulated clients received the $\mathrm{MOH}$ recommended drug regimen for both 
diseases (intervention 10\%; no intervention $3 \%$ ). These proportions increased with a broader category of drugs and dosages deemed correct by the first author and were significantly higher in the intervention outlets $39 \%$ v $18 \% ; \mathrm{p}<0.05)$. For each STI considered alone, the appropriateness of the drugs prescribed was better. For gonorrhoea, $32 \%$ of simulated clients in intervention outlets received the $\mathrm{MOH}$ correct drug and dosage. This proportion increased to $76 \%$ with the broader definition. These two proportions were lower in the no intervention outlets ( $21 \%$ and $64 \%)$. For chlamydia, $12 \%$ of simulated clients in intervention outlets received the $\mathrm{MOH}$ correct drug and dosage. This proportion increased to $41 \%$ with the broader definition. Again, these two proportions were lower in the no intervention outlets ( $5 \%$ and $31 \%$ ).

Well over half the simulated clients who purchased oral medications spent less than 10000 Cedis (about US\$5). In intervention outlets, $17 \%$ of participants spent less than 5000 Cedis and $45 \%$ spent between 5000 and 10000 Cedis. These proportions were similar in the no intervention outlets $(20 \%$ and $40 \%$, respectively).

\section{Discussion}

The training of pharmacists improved the treatment of urethral discharge in Accra, but despite the training less than half the simulated clients received appropriate treatment. More encouraging, the treatment for gonorrhoea was frequently correct without the intervention $(64 \%)$ and improved further in the intervention outlets $(76 \%)$. The treatment for chlamydia was less often appropriate, but again appeared to improve with the training ( $31 \%$ v $41 \%)$. The most recent available data suggest that about $30 \%$ of urethral discharge in Accra is caused by chlamydia. ${ }^{9}$ Most of the counselling and history taking measures also appeared to improve as a result of the training. A notable exception was the promotion of condoms, which was poor in both groups of outlets despite condoms being readily available in most pharmacies.

Strengths of this study include evaluation of a standardised training of pharmacists during a prescribed period of time. The outlets surveyed were randomly selected from a comprehensive sampling frame. Moreover, almost all outlets selected were located and interviewed (96\%). We employed trained simulated clients blinded to the training status of the outlets who completed a standardised questionnaire immediately after each pharmacy encounter.

Our study documents some of the operational, methodological, and statistical difficulties in conducting and evaluating this type of intervention research with limited resources. The major weakness of this study is the low power to detect significant differences because of the small sample size. However, with the exception of the condom counselling, all aspects of STI management were more favourable in the intervention outlets. A further weakness of this study is that outlets were not randomised to receive the training and might systematically differ, so that we cannot exclude the possibility that the results are due to unmeasurable selection bias. Pharmacists were invited to attend the training sessions according to an alphabetical list of outlets.

Moreover, we lacked information on whether the pharmacy staff encountered by the simulated clients had attended the training. Although we evaluated a standardised training curriculum of $\mathrm{MOH}$ recommended drugs, during question and answer sessions alternative drug regimens were discussed and may account for the increase in the correctness of the broader category of drugs. Unfortunately, we do not have information on how often these discussions occurred and what drugs were discussed. Lastly, the small sample size and our uncertainty of the true title of the staff encountered did not permit us to conduct stratified analyses to evaluate whether the position of the staff member encountered was associated with the quality of STI management.

We believe that the differences in STI management measured in the two groups are spuriously low. Some pharmacists in the no intervention outlets may have attended a training workshop at the Ghana Pharmaceutical Society or attended the ongoing WAPTCA training between our sampling and data collection. Unfortunately, we lack information to quantify the information diffusion to no intervention pharmacists through these channels or by means of other less formal channels.

As an extension of the current evaluation, we also conducted a simulated client study at 50 randomly selected outlets in Kumasi, the second largest city in Ghana, before any WAPTCA sponsored training there. Most aspects of STI management were significantly worse compared with the outlets in Accra that had received the training. For example, only $33 \%$ of the simulated clients in Kumasi received acceptable drug provision for gonorrhoea ( $v 76 \%$ Accra intervention; $\mathrm{p}$ value $<0.01$ ) and $8 \%$ received acceptable drug provision for chlamydia ( $v 41 \%$ Accra intervention; $p$ value $<0.01)$. It is possible that pharmacists and staff in Kumasi differ from those in Accra independent of the intervention being evaluated.

Despite its limitations, this study provides some guidance as pharmacist training is expanded to other areas of Ghana. Firstly, the training curriculum needs to be improved, particularly with respect to condom promotion and drug provision for both gonorrhoea and chlamydia when treating urethral discharge. Secondly, the training should not be limited to pharmacists since almost half of all simulated clients were seen by store clerks.

In conclusion, our study has implications to all settings where pharmacies are playing an increasing part in STI management. Only through the appropriate training of all pharmacy staff can we optimise the role of pharmacy outlets in STI management and prevention. Implementing this training is a public health challenge, especially in resource poor countries where the quality of drug provision may remain low despite training. Augmenting training efforts with well designed 
research protocols will help to assess whether STI drug provision by pharmacists is doing more good than harm.

Support for this work was provided with funds from the United States Agency for International Developmer erative agreement No AID/CCP-3079-A-00-5022-00. The views expressed in this document, however, do not necessarily reflect those of the funding agency. We thank Dr Pepin for his review and invaluable input as well as the numerous colleagues review and invaluable input as well as the numerous colleagues throughout this project.

Contributors: The study was conceived by YA-S and MJS; they
throughout this project. developed the study instruments and supervised the data developed the study instruments and supervised the data collection in collaboration with JA. JA supervised data entry and authors collaborated on writing the manuscript.

1 Grosskurth H, Mosha F, Todd J, et al. Impact of improved treatment of sexually transmitted diseases on HIV infection in rural Tanzan
2 Ghana Ministry of Health. Pharmacy outlet study: STD management. Health Research Unit, September, 1996.

3 Crabbe F, Tchupo JP, Manchester T, et al. Prepackaged therapy for urethritis: the "MSTOP" experience in Cameroon. Sex Transm Inf 1998;74:249-52.

4 Garcia PJ, Gotuzzo E, Hughes JP, et al. Syndromic management of STDs in pharmacies: evaluation and randomised intervention trial. Sex Transm Inf 1998;74(Suppl 1): S153-8.

5 Tuladhar SM, Mills S, Acharya S, et al. The role of pharmacists in HIV/STD prevention: evaluation of an STD syndromic management intervention in Nepal. AIDS 1998;12(Suppl 2):S81-7.

6 West Africa Project to Combat AIDS and STD. Evaluation of the quality of treatment of urethral discharge and of genital ulcers offered by pharmacists of Accra and Tema. December, 1998.

7 Centers for Disease Control and Prevention. 1998 Guidesexually transmitted diseases. MMWR 1998:47(RR-1).

8 Ghana Ministry of Health. National reproductive health service protocols. October 1996.

9 Ghana Ministry of Health. STD survey. National AIDS Control Program, 1993. 